\title{
The influence of healthcare provider's autonomy support, autonomous motivation and competence on self-management in kidney transplant patients based on the self-determination theory
}

\author{
Sunyoung Son ${ }^{1}$, Manki Ju${ }^{1}$, Jung Jun Lee ${ }^{1}$, Heeyoung Kim ${ }^{1}$, Mi Kyung $\mathrm{Sim}^{2}$
}

${ }^{1}$ Department of Surgery-Transplantation, Gangnam Severance Hospital, Seoul, Korea
${ }^{2}$ Division of Nursing, Shinsung University, Dangjin, Korea

Background: Continuous follow-ups and self-management are absolutely necessary for transplant patients, but the patients inevitably experience difficulties in carrying out self-management for a lifetime The self-determination theory appears to be very useful in explaining the self-management of transplant recipients, but only a few studies have applied the theory of self-determination to kidney transplant patients in Korea. Thus, this study aimed to confirm the influence of health professionals' autonomy support, renal transplant patients' autonomous motivation and competence on self-management based on the self-determination theory.

Methods: Data were collected from April 20 to August 20, 2020 using self-report questionnaires. The sample was 79 kidney transplant patients who visited outpatient clinics of one general hospital in Seoul, Korea and submitted written consents for voluntary participation in the study. The collected data were analyzed with SPSS ver. 22 for descriptive data analysis and multiple regression analysis.

Results: Self-management was associated with autonomy support of health care professionals, competence, and autonomous motivation. The influential factors on self-management behavior in kidney transplant patients were competence $(\beta=0.377$, $\mathrm{P}<0.01)$ and autonomous motivation $(\beta=0.293, \mathrm{P}<0.01)$, and the explanatory power of these variables were $30.1 \%$.

Conclusions: This study confirmed the effects of autonomous motivation and competence of kidney transplant patients on self-management. Findings indicate that if health care professionals enhance competence and autonomous motivation of patients, self-management can be improved. Thus, health care professionals should improve therapeutic communication with the kidney transplant patients and maintain autonomy support. Various interventional programs by which health care professionals could enhance patients' autonomous motivation and competence should be developed.

Corresponding author: Sunyoung Son

E-mail: otc@yuhs.ac

\footnotetext{
(c) The Korean Society for Transplantation

This is an Open Access article distributed under the terms of the Creative Commons Attribution Non-Commercial License (http://creativecommons.org/licenses/by-nc/4.0/) which permits unrestricted non-commercial use, distribution, and reproduction in any medium, provided the original work is properly cited.
} 